BULL. AUSTRAL. MATH. SOC.

VOL. 7 (1972), 169-182.

\title{
On the units of a modular group ring
}

\section{K.R. Pearson}

It is shown that a finite group $G$ is a normal subgroup of the group of units of the group ring of $G$ over the ring of integers modulo $n$ if and only if $G$ is abelian or $n=2$ and $G$ is isomorphic to the symmetric group on 3 letters.

Let $R$ be a ring with identity $1, G$ a finite group and let $R G$ denote the corresponding group ring. If $\alpha$ is a unit in $R$ and if $g \in G$ then $a g$ is a unit in $R G$, and is called a trivial unit. In particular $\{1 g \mid g \in G\}$ is always a subgroup of the group $(R G)^{*}$ of units of $R G$; by a slight abuse of notation this set will also be denoted by $G$. We consider the following conditions.

I Every unit in $R G$ is trivial.

II Every unit of finite order in $R G$ is trivial.

III Every conjugate in $(R G)^{*}$ of an element of $G$ is trivial; or equivalently, $G$ is a normal subgroup of $(R G)^{*}$.

It is clear that, in general, I $\Rightarrow$ II $\Rightarrow$ III.

For the case $R=Z$, the ring of rational integers, these conditions have been examined by Higman [8] and Berman [2]. Higman showed that I holds if and only if $G$ is either abelian of exponent dividing 4 or 6 or hamiltonian of order a power of 2 . Berman showed that II holds if and only if $G$ is either abelian or hamiltonian of order a power of 2 . In addition, although it is not stated explicitly, his proof shows that (still when $R=Z$ ) II and III are equivalent.

If $R$ has characteristic zero, it is clear that any one of these

Received 12 April 1972. 
three conditions implies the corresponding condition for the case $R=2$. Hence the results of Higman and Berman give some information about the general case when $R$ has characteristic zero.

In this case we consider the conditions when $R=z_{n}$, the ring of rational integers modulo $n$. The results then give some information about the case where $R$ has finite characteristic. Because $Z_{n} G$ is a finite ring it is clear that, when $R=z_{n}$, I and II are equivalent. We prove the following results.

THEOREM 1. Let $G$ be a finite group. $G$ is a normal subgroup of $\left(z_{n} G\right)$ * if and only if $G$ is abelian or $n=2$ and $G \simeq S_{3}$, the symmetric group on 3 letters.

THEOREM 2. Let $G$ be a finite nontrivial group. Every unit in $Z_{n} G$ is trivial if and only if $n=2$ and $|G| \leq 3$ or $n=3$ and $|G|=2$.

A result related to Theorem 1 has been proved in [4] by Eldridge. He has proved that if $G$ is a locally finite $p$-group and $H$ is a subgroup of $G$, then $H$ is normal in $\left(z_{p} G\right)^{*}$ if and only if $H$ is central in G.

51 contains some preliminary results which are perhaps of independent interest. Theorem $I$ is proved in $\$ \$ 2-4$, while Theorem 2 is proved in $\$ 5$.

1. The behaviour of unit groups under ring homomorphisms

Let $R$ be a ring with identity and let $\phi: R \rightarrow S$ be a surjective ring homomorphism. It is easy to see that $\phi$ maps the group $R^{*}$ of units of $R$ into the group $S^{*}$ of units of $S$. That $R^{*} \phi$ is not always the whole of $S^{*}$ may be seen by considering, for example, the canonical homomorphism from $z$ to $z / n Z$. It is of interest to know conditions under which $\phi: R^{*}+S^{*}$ is surjective. When the kernel of $\phi$ is contained in the Jacobson radical of $R$ this is known to be the case (see (2.1) of [6] or Lemma 1 of [5]). The following result shows that it is also the case when $R$ is artinian (irrespective of the kernel of $\phi$ ).

THEOREM 3. Let $R$ be a ring with identity such that $R / J$ is artinian, where $J$ is the Jacobson radical of $R$. If $\phi: R \rightarrow S$ is a 
surjective ring homomorphism then $\phi$ induces a surjective group homomorphism $\phi: R^{*} \rightarrow S^{*}$, where $R^{*}$ and $S^{*}$ denote the group of units of $R$ and $S$ respectively.

Proof. We must show that $\phi$ is onto. Let $k$ denote the kernel of $\phi$.

Suppose firstly that $J=0$. Then $R$ is an internal direct sum

$$
R=R_{1}+\ldots+R_{t} \text {, }
$$

where each $R_{i}$ is a simple artinian ring. By renumbering if necessary we can assume that

$$
K=R_{u+1}+\ldots+R_{t}
$$

Suppose $x \in R$ is such that $x \phi \in S^{*}$; then there is an element $y$ in $R$ such that $x y-1 \in K$ and $y x-1 \in K$. Let

$$
1=e_{1}+\ldots+e_{t},
$$

where $e_{i} \in R_{i}$. Then if

$$
\begin{aligned}
& z=x e_{1}+\ldots+x e_{u}+e_{u+1}+\ldots+e_{t}, \\
& w=y e_{1}+\ldots+y e_{u}+e_{u+1}+\ldots+e_{t},
\end{aligned}
$$

$z w=1=w z$ and $z-x \in K$. Thus $z \in R^{*}$ and $z \phi=x \phi$.

Now consider the general case. Suppose $x \in R$ is such that $x \phi \in S^{*}$. Then $x+K \in(R / K)^{*}$ and so $x+(J+K) \in[R /(J+K)]^{*}$. Since $J \subseteq J+K$ there is a natural homomorphism $\psi: R / J \rightarrow R /(J+K)$. Because $R / J$ is semisimple it follows from the above that there exists $x_{1} \in R$ such that $x_{1}+J \in(R / J) *$ and $x_{1}+(J+K)=x+(J+K)$. By Lemma 1 of [5], there exists $y \in R^{*}$ such that $x_{1}+J=y+J$. Hence there exists $k \in K, j \in J$ such that

$$
x+k=y+j=y\left(1+y^{-1} j\right) \text {. }
$$

But $y^{-1} j \in J$, so $1+y^{-1} j \in R^{*}$ and hence $z=y+j \in R^{*}$. Also $z \phi=x \phi$ since $z-x \in K$. 
COROLLARY 4. Let $R$ be on artinian ring with identity and let $G$ be a finite group ouch that $G \triangleleft(R G)^{*}$. If $P \triangleleft G$ then $(G / P) \triangleleft[R(G / P)]^{*}$.

Proof. Since $R G$ is artinian ([8], Appendix 2, Proposition 6) we can apply the theorem to the homomorphism $\phi: R G \rightarrow R(G / P)$ which extends the identity on $R$, and the canonical homomorphism from $G$ to $G / P$.

COROLLARY 5. If $G \diamond\left(Z_{n}^{G}\right)^{*}$ and $m$ divides $n$ then $G \bullet\left(Z_{m}^{G}\right)^{*}$.

If $R$ is a finite ring

$$
\delta(R)=\left|R^{*}\right| /|R|,
$$

the proportion of invertible elements in $R$, has been considered in [6]. If $\phi: R \rightarrow S$ is a surjective ring homomorphism, it is shown in (3.2) of [6] that $\delta(R)=\delta(S)$ if the kernel of $\phi$ equals the Jacobson radical of $R$.

PROPOSITION 6. Let $R$ be a finite ring and let $\phi: R \rightarrow S$ be a surjective ring homomorphism with kernel $K$. Then $\delta(R)=\delta(S)$ if and only if $K$ is contained in the Jacobson radical of $R$.

Proof. $\phi$ induces a surjective group homomorphism from $R^{*}$ to $S^{*}$ whose kernel is $R^{*} \cap(1+K)$. It is thus easy to see that $\delta(R)=\delta(S)$ if and only if $1+K \subseteq R^{*}$, which in turn is the case if and only if $K$ is a quasi-regular ideal.

\section{Outline the proof of Theorem 1}

If $G$ is abelian, it is clear that $G 4\left(z_{n} G\right)^{*}$. That $S_{3} \triangleleft\left(z_{2} S_{3}\right)^{*}$ is shown in the following lemma. This completes the sufficiency part of Theorem 1 .

LEMMA 7. $S_{3} \triangleleft\left(z_{2} s_{3}\right) *$.

Proof. If $S_{3}=\left\langle a, b \mid a^{2}=b^{3}=1, b a=a b^{2}\right\rangle$ then $\theta: s_{3} \rightarrow z_{2} \oplus M_{2}\left(z_{2}\right)$ given by

$$
a \theta=\left(1,\left(\begin{array}{ll}
0 & 1 \\
1 & 0
\end{array}\right)\right), \quad b \theta=\left(1,\left(\begin{array}{ll}
1 & 1 \\
1 & 0
\end{array}\right)\right)
$$


defines a group homomorphism, and so can be extended to a ring homomorphism $\theta: z_{2} S_{3} \cdot z_{2} \oplus M_{2}\left(z_{2}\right) \cdot \theta$ is onto and its kernel is $J=\{0, \gamma\}$ where $\gamma=1+b+b^{2}+a+a b+a b^{2} \cdot \gamma^{2}=0$ and $J$ is the radical of $2_{2} S_{3}$. By Theorem 3, $\theta$ induces a surjective homomorphism from $\left(z_{2} S_{3}\right)$ * onto GL $\left(2, z_{2}\right)$ whose kernel is $1+J$. Since $\left|\mathrm{GL}\left(2, z_{2}\right)\right|=6$ and since $\theta$ is one-to-one when restricted to $S_{3}$ it follows that $\left(z_{2} S_{3}\right)^{*}=S_{3}(1+J)$. Because $\gamma$ is in the centre of $z_{2} S_{3}$, we see that $\left(z_{2} S_{3}\right) *=S_{3} \times(1+J)$ and $s_{3} \triangleleft\left(z_{2} s_{3}\right) *$.

The necessity part of Theorem 1 remains. Suppose that $G \triangleleft\left(z_{n} G\right)$ * and that $G$ is not abelian. In $\$ \S 3,4$ we consider the case where $n$ is a prime and show that $n=2$ and $G \simeq S_{3}$. In view of Corollary 5 above it follows that $G \simeq S_{3}$ and $n=2^{k}$ for some $k \geq 1$. If $k \geq 2$ and $y=2^{k-1}$. then $(1+y a)^{2}=1$ so that $1+y a$ is a unit of order 2 in $z_{n} S_{3} \cdot$ But

$$
(1+y a) b(1+y a)=b+y a b+y a b^{2} k s_{3} .
$$

Hence $k=1$. This will complete the necessity part.

3.

LEMMA 8. If $p$ is a prime, if $p$ does not divide $|G|$ and if $G \triangleleft\left(z_{p} G\right)^{*}$, then $G$ is abelion.

Proof. For suppose, if possible, that $G$ is not abelian. Since $R=Z_{p} G$ is semisimple, there exists a central idempotent $e$ in $R$ such that $R e \simeq M_{n}\left(\mathrm{GF}\left(p^{k}\right)\right)$ for some $n \geq 2$ and $k \geq 1$. Since $G \triangleleft R^{*}$ it follows that $G e \triangleleft(R e)^{*} \simeq \operatorname{IL}\left(n, p^{k}\right)$. Let $\theta:(R e)^{*} \rightarrow \mathrm{GL}\left(n, p^{k}\right)$ be an isomorphism. Now $p$ divides $\left|\mathrm{SL}\left(n, p^{k}\right)\right|([1]$, Theorem 4.11) so that $\mathrm{SL}\left(n, p^{k}\right) \theta^{-1}$ is not contained in Ge. Since the centre of $\mathrm{GL}\left(n, p^{k}\right)$ is 
contained in the centre of $M_{n}\left(G F\left(p^{k}\right)\right)$ ([1], Theorem 4.8), $G e \subseteq$ centre $(R e) *$ would mean Ge $\subseteq$ centre $(R e)$ and then $R e$ would be commutative since it is spanned by $G e$ over $z_{p} e$. Thus it follows from [1], Theorem 4.9, that $n=2, k=1$ and $p=2$ or 3 .

If $p=2$, we get $|G e|=3$ since $G L(2,2) \simeq S_{3}$. But then Re has dimension at most 3 over $Z_{p} e$ and so cannot be isomorphic to $M_{2}\left(z_{2}\right)$.

Thus $p=3$. Now the only normal subgroup of $\operatorname{GL}(2,3)$ which has order not divisible by 3 and which is not contained in the centre of $\mathrm{GL}(2,3)$ is isomorphic to the quaternion group $H$ of order 8 . Thus $G e \simeq H$. Since $G e$ is a homomorphic image of $G$ it follows from Corollary 4 that $H \triangleleft\left(2_{3} H\right) *$. Let

$$
H=\left\langle i, j \mid i^{2}=j^{2}=t, t^{2}=1, j i=t i j\right\rangle .
$$

Then in $z_{3} H$, if $x=(i+j+i j)(1-t)$ we have $x^{2}=0$ and therefore $1+x$ is a unit with inverse $1-x$. But

$$
(1+x) i(1-x)=1-j-i j-t+t i+t j+t i j k S .
$$

Thus we have a contradiction.

LEMMA 9. If $p$ is a prime $\geq 3$, if $p$ divides $|G|$ and if $G \triangleleft\left(z_{p} G\right)^{*}$, then $G$ is abelian.

Proof. Let $H$ be a p-Sylow subgroup of $G$. We first show that $H$ is in the centre of $G$. For let $g \in G, h \in H$ and let $h$ have order $p^{m}$ with $m \geq 1$. Since $(1-h)^{p^{m}}=1-h^{p^{m}}=0,1-h$ is nilpotent and hence so is $(1-h)^{2}$. Thus $I-(I-h)^{2}=2 h-h^{2}$ is a unit in $2_{p} G$.

Hence there exists $g^{\prime} \in G$ such that $\left(2 h-h^{2}\right) g=g^{\prime}\left(2 h-h^{2}\right)$, or

$$
2 h g-h^{2} g=2 g^{\prime} h-g^{\prime} h^{2} \text {. }
$$

Since $h \neq e, h g$ and $h^{2} g$ are distinct. Thus we get two possibilities, namely 
(i) $g^{\prime} h^{2}=h^{2} g$ and $g^{\prime} h=h g$, in which case $g h=h g$; or

(ii) $2=-1$ (that is, $p=3$ ), $g^{\prime} h=h^{2} g$ and $g^{\prime} h^{2}=h g$, in which case $g^{-1} h g=h^{-1}$.

Suppose, if possible, that $g h \neq h g ;$ then $p=3, g \neq e, g \neq h^{-1}$ and $g h g^{-1}=h^{-1}$. Since $g h g^{-1}=h^{-1}$, it is easy to see that $(1-h) g$ is nilpotent, and so $\alpha=1+(1-h) g$ is a unit. Hence there exists $h^{\prime} \in G$ such that $\alpha h=h^{\prime} \alpha$, which gives

$$
h+g h-h g h=h^{\prime}+h^{\prime} g-h^{\prime} h g .
$$

Since $h, g h$ and $h g h$ are distinct, we have $h ' h g=h g h=g$ and $h^{\prime}=h^{-1}$. Then $h+g h=h^{-1}+h^{-1} g$. Since $h \neq h^{-1}$ we get $h^{-1}=g h$, whence $g=h^{-2}$ and we have a contradiction.

We can now show that $G$ is abelian. For suppose, if possible, that $x, y \in G$ and $x y \neq y x$. If $h \neq 1$ is an element of $H$ then $h$ is in the centre of $G$ and $B=1+(1-h) x$ is a unit. Thus there exists $z \in G$ such that $\beta y=z \beta$, and so

$$
y+x y-h x y=z+z x-h z x .
$$

Since $x y \neq y x, y \neq e$ and $x \neq h^{-1}$ so that $y, x y$ and hxy are distinct. Thus hxy $h y z x$ and $z=x y x^{-1}$. Now $y+x y=x y x^{-1}+x y$, which means $y=x y x^{-1}$ or $x y=y x$.

\section{4. $n=2$ and $|G|$ is even}

We are left with $G \triangleleft\left(Z_{2} G\right) *$ and $|G|$ even. We show that either $G$ is abelian or $G \simeq S_{3}$.

In what follows we will often have a situation similar to the following. Suppose

$$
x_{1}+\ldots+x_{n}=y_{1}+\ldots+y_{n},
$$

where $x_{i}, y_{i} \in Z_{2} G$ and $x_{1}, \ldots, x_{n}$ are distinct. Then the $y_{i}$ must be a permutation of the $x_{j}$ and so this leads to $n$ ! possible cases. 
LEMMA 10. If $h \in G$ has order $2^{m}$ with $m \geq 2$ then $h$ is in the centre of $G$.

Proof. Let $\theta \in G$. Since $I+(1+h) h$ is a unit, there exists $z \in G$ with $\left(1+h+h^{2}\right) g=z\left(1+h+h^{2}\right)$. This gives $g h g^{-1}=h^{8}$ where $\boldsymbol{a}= \pm 1$. Thus $(1+h) g$ is nilpotent and so there exists $w \in G$ with

$$
[1+(1+h) g] g=w[1+(1+h) g] \text {. }
$$

If $g h \neq h g$ then $g h g^{-1}=h^{-1}$ and this leads to a contradiction.

LEMMA 11. If any two elements of ordex 2 in $G$ commute then $G$ is abelian.

Proof. Let $b \in G$ have order 2 . We show that $b$ is in the centre of $G$. For let $g \in G$. If for $x \in G, b^{x}$ denotes $x b x^{-1}$ then, for all $t \geq 1$,

$$
((1+b) g)^{t}=(1+b)\left(1+z^{g}\right) \ldots\left(1+z^{g^{t-1}}\right) g^{t}
$$

There exists an integer $n$ such that $g^{n}=1$ and so

$$
((1+b) g)^{n+1}=(1+b)\left(1+b^{g}\right) \ldots\left(1+b^{g^{n-1}}\right)(1+b) g^{n+1}=0,
$$

since $b, b^{g}, \ldots, b^{g^{n-1}}$ all commute and $(1+b)^{2}=0$. Thus $\alpha=1+(1+b) g$ is a unit and there exists $h \in G$ with $\alpha g=h \alpha$. Consideration of the six cases gives $g b=b g$.

Suppose $x, y \in G$ and $x y \neq y x$. Then let $b \in G$ have order 2 . Since $B=1+(1+b) x$ is a unit, there exists $z \in G$ with $\beta y=z \beta$ and this yields $y x=b x y$. Now $y^{x^{2}}=y$ and $y^{x} \neq y$ so that $y^{x^{n}}=y$ if and only if $n$ is even. Thus $x$ has even order, say $2^{8} t$ where $t$ is odd and $s \geq 1$. Then if $z=x^{t}, z$ has order $2^{s}$ and $y z=b^{t} x^{t} y=b z y$. Now if $z \geq 2$ this contradicts Leme 10 while if $\theta=1$ this contradicts the paragraph above. Hence $G$ is abelian. LEMMA 12. If $x, y \in G$ both have order 2 and if $x y \neq y x$ then 
xy has order 3 .

Proof. Let $x y=b$ and let $b$ have order $m$. Then $(b, x)$ is a dihedral group of degree $m$ and $m \geq 3$. Now $((1+x) b(1+x))^{2}=0$ and so $\beta=1+(1+x) b(1+x)$ is a unit. Thus there exists $g \in G$ such that $\beta b=g^{\beta}$. Since $g \in\langle b, x\rangle$ and $g$ has the same order as $b$, it follows from a knowledge of the dihedral group that $g=b^{j}$ for some $j$. From $B b=b^{j} \beta$ we get $x b^{2}+x=x b^{-1-j}+x b^{1-j}$. If $b^{1-j}=1$ we get $b^{4}=1$, which contradicts Lemma 10 , and so $b^{1-j}=b^{2}$. If we substitute this in $\beta b=b^{j} \beta$ we get $b^{3}=1$ and $m=3$.

LEMMA 13. Let $a \in G$ have order 2 and suppose there exists $b \in G$ of order 2 such that $a b \neq b a$. If $c \in G$ has order 2 then $a c \neq c a$.

Proof. For suppose $a c=c a$; then $(a+c)^{2}=0$ and there exists $d \in G$ such that

$$
(1+a+c) a b=d(1+a+c),
$$

and this leads to a contradiction.

COROLLARY 14. Let $a, b \in G$ both have order 2 with $a b \neq b a$. If $c, d \in G$ both have order 2 then $c d \neq d c$.

Proof. By the lemma, $a c \neq c a$. Then from the lemma with $a, b, c$ replaced by $c, a, d$ respectively we get $c d \neq d c$.

LEMMA 15. If $G$ is not abelian then $|G|$ is not divisible by 4 .

Proof. If 4 divides $|G|$ then $G$ contains a subgroup of order 4. This cannot be cyclic, by Lemma 10, so contains two commuting elements of order 2 . It then follows from Corollary 14 and Lemma 11 that $G$ is abelian.

LEMMA 16. Suppose $G$ is not abelicon. Then $G$ contains two elements $a, b$ of order 2 such that $a b \neq b a$. The only elements of order 2 in $G$ are $a, b$, and $a b a$ cond, if $K$ is the subgroup generated by these elements of order 2 , then $K=\{1, a, b, a b a, a b, b a\}$ and $K \simeq S_{3}$.

Proof. The existence of $a$ and $b$ is given by Lemma 11 . We know 
from Lemma 12 that $a b$ has order 3 and hence $a b a$ has order 2 . Suppose that $c$ is an element of order 2 which is distinct from $a, b$ and $a b a$, and let $d=a b, f=a c$ and let $H$ be the subgroup generated by $d$ and $f$; we know that $d$ and $f$ have order 3 . Also $d f=(a b a) c, d^{2} f=b c, d f^{2}=a(b c) a, d^{2} f^{2}=b(a c a)$ all have order 3. Thus, for all $i, j$,

$$
f^{j} d^{i} f^{j}=d^{-1} f^{-j} d^{-i}
$$

It now follows as on page 321 of [7] that any element of $H$ can be written as $d^{i}, d^{i} f d^{j}, d^{i} f^{-1} d^{j}$ or $d^{i} f d^{j} f^{-1} d^{k}$. It can then be verified by using (1) that $H$ has exponent 3 . Hence $H$ is abelian, by Lemma 8 , and $d f=f d$. If $x=1+(1+d)\left(1+f+f^{2}\right)$ then $x^{3}=1$ and $b x b=x^{2}$, which gives $x b x^{-1}=b x$ and means that $x \in G$. Since $f$, $d f$ and $d f^{2}$ are distinct, $x$ must then equal one of them, and this yields $f=d$ or $d^{2}$, which in turn yields $c=b$ or $a b a$ and is a contradiction.

It is routine to verify that $K$ is as stated and is isomorphic to $S_{3}$

In what follows we assume $G$ is nonabelian. Let $N$ be the radical of $S=Z_{2} G$, let $\phi: S \rightarrow S / N=\bar{S}$ be the canonical map, let

$$
\bar{S}=\bar{S}\left(e_{1} \phi\right)+\ldots+\bar{S}\left(e_{t} \phi\right),
$$

where the $e_{i} \phi$ are central primitive orthogonal idempotents in $\bar{S}$ and let

$$
\bar{S}\left(e_{i} \phi\right) \simeq M_{n_{i}}\left(G F\left(2^{k} i\right)\right) \text {. }
$$

\section{LEMMA 17.}

(i) At least one $n_{i} \geq 2$.

(ii) If $n_{i} \geq 2$ then $n_{i}=2, k_{i}=1$ and

$$
(G \phi)\left(e_{i} \phi\right)=\left(\bar{S}\left(e_{i} \phi\right)\right)^{*} \simeq \mathrm{GL}(2,2) \text {. }
$$

Proof. (i) We know from Theorem 3 that $\phi: S^{*} \rightarrow \bar{S}^{*}$ is onto and has kernel $I+D$. If $g \in G \cap(I+N)$ then $1+g \in N$ and so 
$(1+g)^{2^{k}}=1+g^{2^{k}}=0$ for some $k$; hence $g$ has order 2 by Lemma 16 . But $1+a k N$, since otherwise $(1+a) b=b+a b \in N$, and this is impossible because $(b+a b)^{3}=(b+a b)^{2} \neq 0$. Similarly $1+b \neq N$ and $1+a b a \notin N$. Thus $G \cap(1+N)=\{1\}$ and $G \phi \simeq G$. Hence $\bar{S}$ is not commutative, so at least one $n_{i} \geq 2$.

(ii) Suppose $n_{i} \geq 2$. Now $(G \phi)\left(e_{i} \phi\right) \triangleleft\left(\bar{S}\left(e_{i} \phi\right)\right) *$ and, since $\bar{S}\left(e_{i} \phi\right)$ is spanned by $(G \phi)\left(e_{i} \phi\right)$ over $z_{2},(G \phi)\left(e_{i} \phi\right)$ is not contained in the centre of $\left(\bar{S}\left(e_{i} \phi\right)\right) *$. If $n_{i} \geq 3$ or if $n_{i}=2$ and $k_{i}>1$ it follows from Theorem 4.9 of [1] that $(G \phi)\left(e_{i} \phi\right)$ contains a subgroup $H$ with $H \simeq \operatorname{SL}\left(n_{i}, 2^{k}\right)$ but in this case 4|| $\operatorname{Sic}\left(n_{i}, 2^{k}\right) \mid$ ([1], Theorem 4.11), and this contradicts Lemma 15. Thus $n_{i}=2$ and $k_{i}=1$. Since $S\left(e_{i} \phi\right)$ is spanned by $(G \phi)\left(e_{i} \phi\right)$ over $z_{2}$ and has dimension 4 , it follows that $(G \phi)\left(e_{i} \phi\right)=\left(S\left(e_{i} \phi\right)\right)^{*}$, since otherwise $\left|(G \phi)\left(e_{i} \phi\right)\right| \leq 3$.

LEMMA 18. $G$ is an intemal direct product $G=K \otimes L$ for some abelian group $L$ of odd order.

Proof. Let $\psi_{i}: \bar{S} \rightarrow \bar{s}\left(e_{i} \phi\right)$ be given by $\bar{s} \psi_{i}=\bar{s}\left(e_{i} \phi\right)$, and let $L_{i}$ be the kernel of $\phi \psi_{i}$.

Suppose $n_{i}=2$. Since $G \phi \psi_{i} \simeq S_{3}$ and 4 does not divide $|G|$, $\left|L_{i}\right|$ must be odd. Hence $L_{i} \cap K=\{1\}$ or $\langle a b\rangle$.

Suppose that $L_{i} \cap K=\langle a b\rangle$ for all $i$ such that $n_{i}=2$. Then if $n_{i}=2$,

$$
b \phi \psi_{i}=(a \cdot a b) \phi \psi_{i}=(a \cdot 1) \phi \psi_{i}=(1 \cdot a) \phi \psi_{i}=(a b a) \phi \psi_{i} .
$$

Also, if $n_{j}=1$, then, since $S \phi \psi_{j}$ is commutative,

$$
b \phi \psi_{j}=(a \cdot a b) \phi \psi_{j}=(a b a) \phi \psi_{j}
$$

Thus $b \phi \psi_{i}=a b a \phi \psi_{i}$ for all $i$, which means that $b \phi=a b a \phi$ and 
contradicts the fact that $\phi$ is one-to-one on $G$ (see the proof of $(i$ ), Lemma 17).

Thus for some $i, n_{i}=2$ and $L_{i} \cap K=\{1\}$. Since $L_{i}$ and $K$ are both normal in $G$, and since

$$
|G|=\left|L_{i}\right|\left|\left(\bar{S}\left(e_{i} \phi\right)\right) *\right|=\left|L_{i}\right||K|,
$$

we must have $G=K \otimes L_{i}$. Further, it follows from Lemma 8 that $L_{i}$ is abelian.

LEMMA 19. $G=K$.

Proof. Since $L$ is abelian and of odd order, $Z_{2} L$ is isomorphic to a direct sum of fields $F_{1} \oplus \ldots \oplus F_{t}$. Then

$$
Z_{2} G=\left(Z_{2} L\right) K \simeq\left(\bigoplus_{i=1}^{t} F_{i}\right)(K) \simeq \bigoplus_{i=1}^{t}\left(F_{i} S_{3}\right)=M,
$$

say. Now if $N_{i}$ the radical of $F_{i} S_{3}$, then, as in Lemma 7 , $E_{i} S_{3} / N_{i} \simeq F_{i} \oplus M_{2}\left(F_{i}\right)$. Thus if $J$ is the radical of $M$, then

$$
M / J \simeq \bigoplus_{i=1}^{t}\left\{F_{i} \oplus M_{2}\left(F_{i}\right)\right\}
$$

It now follows from Lemma 17 that $F_{i} \simeq z_{2}$ for all $i$. But then $z_{2} L$ is isomorphic to $t$ copies of $z_{2}$ and $|L|=1$.

\section{Proof of Theorem 2}

Suppose that every unit in $Z_{n} G$ is trivial. It follows from Theorem 1 that either $G$ is abelian or else $n=2$ and $G=S_{3}$. In the latter case, if $\gamma$ is the sum of all the elements of $G$ then $\gamma^{2}=0$ so $(1+\gamma)(1-\gamma)=1$ and $1+\gamma$ is a non-trivial unit. Thus $G$ is abelian.

We next notice that if $m$ divides $n$ then every unit in $Z_{m} G$ is trivial. For let $\theta: Z_{n} G \rightarrow Z_{m} G$ be the homomorphism extending the canonical homomorphism from $Z_{n}$ to $Z_{m}$ and the identity on $G$. Then if 
$B$ is a unit in $Z_{m} G$, it follows from Theorem 3 that there is a unit $\alpha$ in $Z_{n} G$ such that $\alpha \theta=\beta$. Since $\alpha$ is trivial, $\beta$ must be also.

Let $p$ be a prime dividing $n$. Notice that $p^{2}$ does not divide $n$, for otherwise, if $\gamma$ is the sum of all the elements of $G$ then $[(n / p) \gamma]^{2}=0$ and so $1+(n / p) \gamma$ is a non-trivial unit.

Let $H$ be a subgroup of $G$ of order $k$; then $Z_{p} H$ has only trivial units.

If $p$ divides $k$ and if $\gamma$ is the sum of all the elements in $H$ then $r^{p}=0$ in $Z_{p} H$ so that $I+r$ is a unit. Because it is non-trivial if $k>2$, we must have $p=k=2$.

If $p \neq k$ and if $k$ is a prime we know from Theorem 4.7 of [3] that $z_{p} H \simeq z_{p} \oplus\left\{[(k-1) / \mu]\right.$ copies of $\left.G F\left(p^{\mu}\right)\right\}$

where $\mu$ is the order of $p$ moduio $k$. Thus $z_{p} H$ has

$$
(p-1)\left(p^{\mu}-1\right)[(k-1) / \mu]
$$

units. But $z_{p} H$ has only $(p-1) k$ trivial units. Since $\mu$ divides $k-1$ by Fermat's Theorem and $k$ divides $p^{\mu}-1$, we must have $\mu=k-1$ and $p^{k-1}=k$. This latter equation means that either $p=2$ and $k=3$, or $p=3$ and $k=2$.

Firstly consider what happens if $p=3$; then $G$ must be a 2-group. But, again using Theorem 4.7 of [3], $Z_{3} C_{4} \simeq 2 Z_{3} \oplus \mathrm{GF}(9)$ has 32 units and only 8 trivial units, while $z_{3}\left(C_{2} \times C_{2}\right)=4 Z_{3}$ has 16 units and only 8 trivial units. Thus $G$ must be of order 2 . Also, in $z_{6} C_{2},(3+3 x)^{2}=0$ so $1+(3+3 x)$ is a non-trivial unit; thus $n=3$.

The only remaining possibility is that $n=2$. But, again using Theorem 4.7 of [3], $z_{2} C_{9} \simeq z_{2} \oplus \mathrm{GF}(4) \oplus \mathrm{GF}(64)$ has 189 units and only 9 trivial ones while $z_{2}\left(C_{3} \times C_{3}\right)=z_{2} \oplus 4 \mathrm{GF}(4)$ has 243 units but only 
9 trivial units. Thus $G$ must be cyclic of order 2 or 3 .

Conversely, it is easily checked that if $n=2$ and $|G| \leq 3$ or if $n=3$ and $|G|=2$ then $Z_{n} G$ has only trivial units.

\section{References}

[1] E. Artin, Geometric algebra (Interscience, New York, London, 1957).

[2] С.Д. Берман [S.D. Berman], "Об уравненин $x^{m}=1$ в целочнсленном групповом кольце" [On the equation $x^{m}=1$ in an integral group ring], Ukrain. Mat. Ž. 7 (1955), 253-261.

[3] Charles Allen Cable, "On the decomposition of a group ring", (PhD dissertation, Pennsylvania State University, University Park, Pennsylvania, 1969).

[4] Klaus E. Eldridge, "On normal subgroups in modular group algebras", (unpublished).

[5] Klaus E. Eldridge and Irwin Fischer, "D.C.C. rings with a cyclic group of units", Duke Math. J. 34 (1967), 243-248.

[6] H.K. Farahat, "The multiplicative groups of a ring", Math. 2. 87 (1965), 378-384.

[7] Marshall Hall, Jr, The theory of groups (The Macmillan Company, New York, 1959).

[8] Graham Higman, "The units of group-rings", Proc. London Math. Soc. (2) $46(1940), 231-248$.

[9] J. Lambek, Lectures on mings and moduZes (Blaisdeli, Waltham, Massachusetts; 1966$)$.

Department of Mathematics,

La Trobe University,

Bundoora,

victoria. 